EPJ Web of Conferences 113,05012 (2016)

DOI: $10.1051 /$ epjconf/201611305012

C) Owned by the authors, published by EDP Sciences, 2016

\title{
Sea Quarks in the Proton
}

\author{
Paul E Reimer ${ }^{1, a}$ and the Fermilab SeaQuest Collaboration \\ ${ }^{1}$ Physics Division, Argonne National Laboratory, Argonne, IL 60439 USA
}

\begin{abstract}
The proton is a composite particle in which the binding force is responsible for the majority of its mass. To understand this structure, the distributions and origins of the quark-antiquark pairs produced by the strong force must be measured. The SeaQuest collaboration is using the Drell-Yan process to elucidate antiquark distributions in the proton and to study their modification when the proton is held within a nucleus.
\end{abstract}

Over the last half century, an understanding of the proton has developed in which it contains three valence quarks and an effervescing sea of antiquarks, quarks and gluons. The flavors of the three valence quarks determine many of the characteristics, but the strong force and the sea of antiquarkquark pairs are primarily responsible for its mass. Experimentally, this picture was established based on a variety of probes, one of the most important being deep inelastic scattering (DIS). DIS, however, lacks the ability to distinguish between antiquarks and quarks.

Measurements of the sea quarks are important, however, as they are manifestations of the effects of the strong force. The Drell-Yan reaction necessarily involves antiquarks. To leading order in the strong coupling constant, $\alpha_{s}$, the Drell-Yan process is the annihilation of a quark from one hadron with an antiquark from another hadron into a massive virtual photon that decays into a detectable leptonantilepton pair, a process first observed by J.H. Christenson et al. [1]. The features of the cross section were explained by S.D. Drell and T.-M. Yan [2] in terms of the parton model as a hard scattering of point-like particles multiplied by a convolution of the parton distributions of the interacting hadrons:

$$
\frac{d^{2} \sigma}{d x_{1} d x_{2}}=\frac{4 \pi \alpha_{e}^{2}}{9 s x_{1} x_{2}} \sum_{q \in\{u, d, s, \ldots\}} e_{q}^{2}\left[\bar{q}_{1}\left(x_{1}\right) q_{2}\left(x_{2}\right)+q_{1}\left(x_{1}\right) \bar{q}_{2}\left(x_{2}\right)\right],
$$

where $x_{1(2)}$ represent Bjorken-x, $x_{\mathrm{Bj}} ; q_{i}\left(x_{i}\right)$ is the parton distribution of quark of flavor $q$; $e_{q}$ is the charge of quark flavor $q$; $\sqrt{s}$ is the center-of-mass energy; $\alpha_{e} \approx 1 / 137$ is the fine structure constant; and the sum is over all quark flavors. The $e_{q}^{2}$ weighting of the parton distributions implies that the cross section is primarily sensitive to the $u$ - and $\bar{u}$-quark distributions. This expression is only leading order, and next-to-leading order (NLO) terms in $\alpha_{s}$ contribute up to half of the cross section. In a typical fixed-target experiment, the acceptance is biased toward large Feynman- $x, x_{\mathrm{F}} \approx x_{1}-x_{2}$, and thus the beam parton is generally a large $x_{\mathrm{Bj}}$ valence parton. For a proton beam this implies that the interaction is between a valence beam quark and a lower- $x_{\mathrm{Bj}}$ target anti-quark.

The SeaQuest experiment was proposed to measure the distributions of sea quarks in the nucleon and the modifications of these distributions in nuclei. The experiment is designed to measure the $\mu^{+} \mu^{-}$(dimuon) decay of the Drell-Yan virtual photon, using a $120 \mathrm{GeV}$ proton beam extracted in a

\footnotetext{
ae-mail: reimer@anl.gov
} 


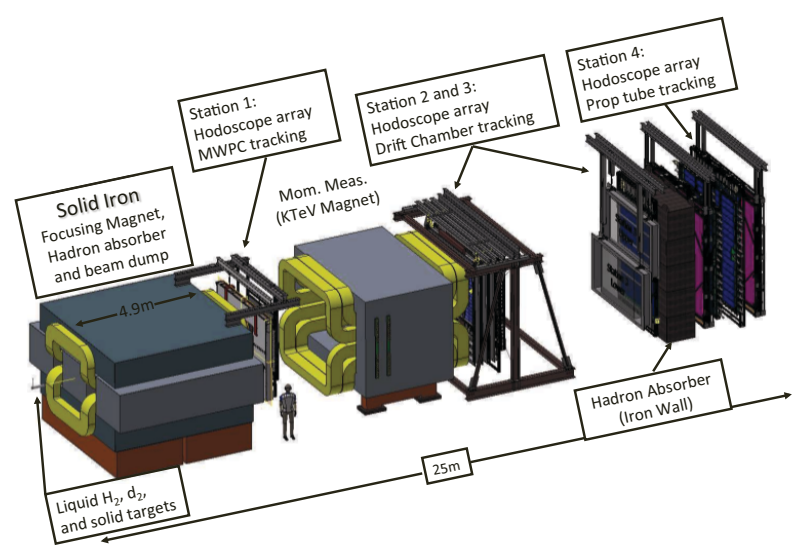

Figure 1. This figure schematically shows the SeaQuest spectrometer. The $120 \mathrm{GeV}$ proton beam enters from the left, and the solid iron magnet also serves as an absorber for the beam that did not interact in a target.

$4 \mathrm{~s}$ long, slow-spill from the Fermilab Main Injector. The spectrometer's basic concept is based on previous Fermilab Drell-Yan spectrometers that were used with $800 \mathrm{GeV}$ extracted beams [3-5].To account for the difference in boost, the spectrometer was shortened significantly. Key features of the spectrometer include two large dipole magnets and four independent tracking/triggering stations. A schematic drawing of the spectrometer is shown in Fig. 1. More details about the spectrometer may be found in Ref. [6]. The experiment uses liquid hydrogen, liquid deuterium, carbon, iron and tungsten targets as well as an empty liquid target flask and a "no target" position for background subtraction. These targets are mounted on a horizontally movable table, so that only one target intercepts the beam during any given slow-extraction spill. The first dipole is a closed-aperture, solid iron magnet. The beam which does not interact in the targets is absorbed in the iron of the first magnet, allowing only (to a large extent) muons to traverse the remaining spectrometer. The downstream magnet is a large open-aperture magnet that was previously used in the Fermilab KTeV experiment. Each of the tracking/triggering stations consisted of a set of scintillator hodoscopes that provided fast information for an FPGA-based road look up and a drift chamber for fine-grained tracking.

Initially, it was believed that, at some very low energy scale, the proton could be described by empirically derived distributions of three valence quarks [7]; however, this approach disagreed with existing data [8]. Gluons spontaneously splitting into antiquark-quark pairs and the spontaneous recombination of antiquark-quark pairs into gluons was believed to be the process primarily responsible for the generation of the quark sea. Studying the flavor dependence of the antiquark sea can determine the relevant importance of this process. Nevertheless, the assumption that the sea was flavor symmetric was maintained until measurements by NMC [9] of the Gottfried sum rule [10] showed otherwise. Because of its sensitivity to the anti-quarks, Martin suggested that a comparison of proton-proton to proton-deuterium Drell-Yan would be an excellent method to study this difference [11]. Using the approximations that the Drell-Yan cross section is dominated by $u_{\text {beam }}$ and $\bar{u}_{\text {target }}$ annihilations and that the proton and neutron are charge symmetric, the approximate sensitivity is:

$$
\frac{\sigma^{\mathrm{pd}}}{2 \sigma^{\mathrm{pp}}} \approx \frac{1}{2}\left(1+\frac{\bar{d}}{\bar{u}}\right),
$$



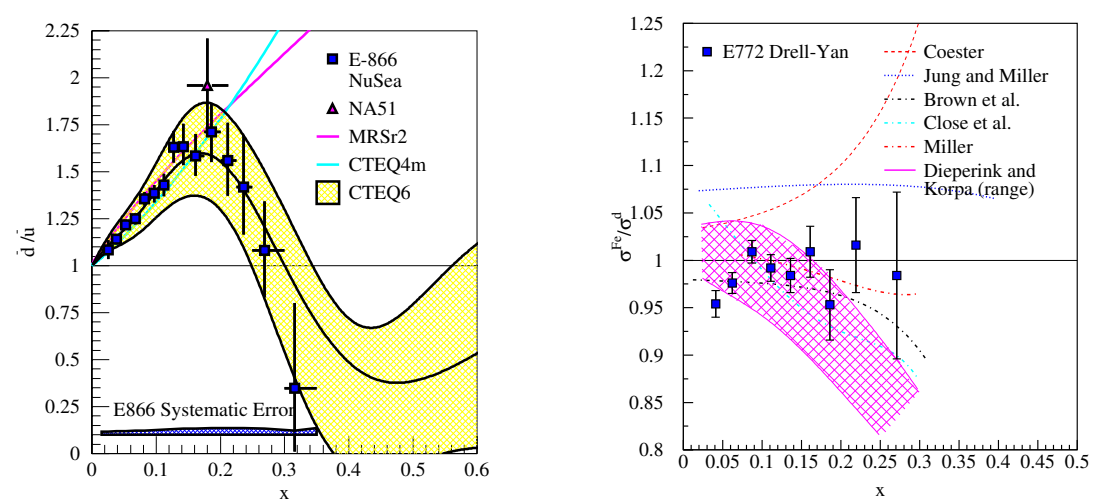

Figure 2. On the left, this figure shows the CERN NA51 [12] and Fermilab E866/NuSea [5] measurements of the $\bar{d} / \bar{u}$ ratio using Drell-Yan. The yellow band indicates a global fit by CTEQ including these data [13]. The right figure shows the lack of any nuclear dependence in the sea quarks as measured by Fermilab E-772, along with a model prediction [16] and several postdictions [17].

where $\sigma^{\mathrm{pp}(d)}$ is the proton (deuterium) cross section and $\bar{d}(\bar{u})$ is the antidown (antiup) quark distribution. This idea was seized upon by the CERN NA51 [12] and the Fermilab E-866/NuSea [5] experiments, whose results are shown in Fig. 2. The Fermilab NuSea data revealed a surprising trend in the flavor asymmetry-returning to unity as $x_{\mathrm{Bj}}$ increases-but with decreasing statistical precision at larger $x_{\mathrm{Bj}}$. The Fermilab E-906/SeaQuest experiment will measure this ratio up to $x_{\text {target }} \approx 0.45$. After engineering runs for both the SeaQuest spectrometer and the high-duty factor, slow-spill extraction of protons from the Main Injector, SeaQuest began collecting production data in the spring of 2014. SeaQuest anticipates to continue recording data until the summer of 2016.

The residual strong force is responsible for holding the positively-charged nucleus of an atom together. Evidence from DIS [14] has shown that the distributions of quarks and antiquarks within the individual nucleons are modified, presumably because of this. The modification of the sea quark distributions may be completely different, but DIS is much less sensitive to this. In Fermilab E-772 using the Drell-Yan reaction, no modifications to sea quark distributions were observed [4]. (See Fig. 2.) Models of nuclear binding that explain the EMC effect rely on the exchange of virtual mesons [15]. Based on these models, significant enhancements of the antiquark distributions in nuclei were expected [16]. The lack of sea-quark nuclear effects prompted a number of new models [17]. For $x>0.2$, the E-772 statistical uncertainties allow significant freedom for these models.

The SeaQuest spectrometer is a general purpose muon spectrometer. Several future measurements using the SeaQuest spectrometer have been proposed to and approved by Fermilab. These include plans for a polarized hydrogen target to explore the Sivers asymmetry, $f_{1}^{\perp}\left(x, k_{T}\right)$, in the sea quarks and its relation to orbital angular momentum. The polarized target is being provided by Los Alamos collaborators and work on the beam line for better collimation is underway at Fermilab. The target will be installed immediately after the completion of the unpolarized experiment, starting in summer, 2016 [18]. An interesting property of the Sivers asymmetry is that its sign is probe dependent. That is, $\left.f_{1}^{\perp}\left(x, k_{T}\right)\right|_{\text {DIS }}=-\left.f_{1}^{\perp}\left(x, k_{T}\right)\right|_{\text {DY }}$. This property has yet to be tested, primarily due to a lack of polarized Drell-Yan data in a the same kinematic region as the existing DIS data. This can be remedied by using a polarized proton beam on an unpolarized target in SeaQuest, as proposed in Fermilab E-1027 [19]. 
A recent and very exciting addition to the SeaQuest physics program is a search for dark photons and dark Z's. If long-lived dark photons are produced by the proton-nucleus collisions in the iron of the first magnet, they may be identified by selecting dimuons with a vertex downstream of the first magnet and searching for a peak in the reconstructed dimuon mass spectrum [20].

The SeaQuest experiment was initially designed to measure unpolarized sea quark distributions to elucidate the origins of the sea quarks and the modifications of their distributions in a nuclear environment. The physics program has vastly expanded since then to include searches for dark sector particles and measurements of the Sivers asymmetry with polarized targets and beams. The experiment has been commissioned and is collecting data. The unpolarized phase is expected to be complete in the summer of 2016, after which the installation of a polarized target will begin.

\section{Acknowledgements}

This work is supported in part by the U.S. Department of Energy, Office of Science, Office of Nuclear Physics, under Contract No. DE-AC02-06CH11357.

\section{References}

[1] J.H. Christenson, et al. Phys. Rev. Lett. 25, 1523 (1970); J.H. Christenson, et al. Phys. Rev. D 8, $2016(1973)$

[2] S.D. Drell, T.M. Yan, Phys. Rev. Lett. 25, 316 (1970); S.D. Drell, T.M. Yan, Phys. Rev. Lett. 25, $902(1970)$

[3] G. Moreno, et al. Phys. Rev. D 43, 2815 (1991)

[4] D.M. Alde, et al., Phys. Rev. Lett. 64, 2479 (1990)

[5] E.A. Hawker et al. (Fermilab E866/NuSea), Phys. Rev. Lett. 80, 3715 (1998); R.S. Towell et al. (Fermilab E866/NuSea), Phys. Rev. D64, 052002 (2001)

[6] The SeaQuest spectrometer at Fermilab (2016), in preparation

[7] M. Glück, R. Godbole, E. Reya, Z. Phys. C 41, 667 (1989)

[8] M. Glück, E. Reya, A. Vogt, Z. Phys. C 53, 127 (1992)

[9] P. Amaudruz, et al. Phys. Rev. Lett. 66, 2712 (1991)

[10] K. Gottfried, Phys. Rev. Lett. 18, 1174 (1967)

[11] A. Martin, W. Stirling, R. Roberts, Physics Letters B 308, 377 (1993)

[12] A. Baldit et al. (NA51), Phys. Lett. B332, 244 (1994)

[13] J. Pumplin, et al., Journal of High Energy Physics 2002, 012 (2002)

[14] J.J. Aubert et al. (European Muon), Phys. Lett. B123, 275 (1983)

[15] J. Carlson, R. Schiavilla, Rev. Mod. Phys. 70, 743 (1998)

[16] F. Coester (2001), private communication

[17] H. Jung, G.A. Miller, Phys. Rev. C41, 659 (1990); G.E. Brown, M. Rho, Nucl. Phys. A590, 527c (1995); J.R. Smith, G.A. Miller, Phys. Rev. Lett. 91, 212301 (2003) A.E.L. Dieperink, C.L. Korpa, Phys. Rev. C55, 2665 (1997)

[18] A. Klein, X. Jiang et al. (Fermilab E1039/SeaQuest) (2013), Fermilab Proposal 1039 http://www.fnal.gov/directorate/program_planning/June2013PACPublic/P1039_LOI_polarized_DY.pdf

[19] W. Lorenzon, P.E. Reimer et al. (Fermilab E1027/SeaQuest) (2012), Fermilab Proposal 1027 https://seaquest-docdb.fnal.gov:cgi-bin/ShowDocument?docid=535

[20] S. Gardner, R.J. Holt, A.S. Tadepalli, Phys. Rev. D (2015), in preparation 